\title{
Alcohol consumption impairs hepatic protein trafficking: mechanisms and consequences
}

\author{
Blythe D. Shepard • David J. Fernandez • \\ Pamela L. Tuma
}

Received: 23 July 2009/Accepted: 11 October 2009/Published online: 5 November 2009

(C) Springer-Verlag 2009

\begin{abstract}
Alcoholic liver disease is a major biomedical health concern in the United States. Despite considerable research efforts aimed at understanding the progression of the disease, the specific mechanisms leading to alcohol-induced damage remain elusive. Numerous proteins are known to have alcohol-induced alterations in their dynamics. Defining these defects in protein trafficking is an active area of research. In general, two trafficking pathways are affected: transport of newly synthesized secretory or membrane glycoproteins from the Golgi to the basolateral membrane and clathrin-mediated endocytosis from the sinusoidal surface. Both impaired secretion and internalization require ethanol metabolism and are likely mediated by acetaldehyde. Although the mechanisms by which ethanol exposure impairs protein trafficking are not fully understood, recent work implicates alcohol-induced modifications on tubulin or components of the clathrin machinery as potential mediators. Furthermore, the physiological ramifications of impaired protein trafficking are not fully understood. In this review, we will list and discuss the proteins whose trafficking patterns are known to be impaired by ethanol exposure. We will then describe what is known about the possible mechanisms leading to impaired protein trafficking and how disrupted protein trafficking alters liver function and may explain clinical features of the alcoholic patient.
\end{abstract}

Keywords Clathrin - Endocytosis - Ethanol · Hepatotoxicity $\cdot$ Secretion $\cdot$ Tubulin

Blythe D. Shepard and David J. Fernandez contributed equally to this work.

B. D. Shepard · D. J. Fernandez · P. L. Tuma $(\bowtie)$

Department of Biology, The Catholic University of America,

620 Michigan Avenue, NE, Washington, DC 20064, USA

e-mail: tuma@cua.edu

\section{Introduction}

For over 30 years, defining the alcohol-induced defects in protein trafficking has been an active area of research in understanding liver injury. To date, numerous proteins are known to have alcohol-induced alterations in their dynamics. In general, two transport pathways appear to be affected: transport of newly synthesized secretory or membrane glycoproteins from the Golgi to the basolateral membrane and clathrin-mediated endocytosis from the sinusoidal surface. In this review, we will first list and discuss hepatic proteins whose trafficking is known to be impaired by ethanol exposure. We will then describe our current understanding of the mechanisms and physiological consequences of impaired protein trafficking.

\section{Ethanol-induced impairment of biosynthetic protein trafficking}

Over three decades ago, it was reported that ethanol exposure led to defects in protein secretion. Since then, many researchers have confirmed these results in numerous model systems by monitoring bulk secretion with radiolabeled proteins or by monitoring the fates of specific molecules (Table 1). These early studies also determined that plasma membrane delivery of newly synthesized transmembrane proteins was impaired upon ethanol exposure leading to altered surface composition (Table 2). In this section, we will briefly describe these early experiments and highlight some important findings.

Ethanol-induced impairment of constitutive secretion

The liver synthesizes the majority of serum proteins and lipoproteins and is characterized by robust constitutive 
Table 1 Ethanol-induced defects in secretion

\begin{tabular}{llll}
\hline Protein/label & EtOH exposure & System & References \\
\hline${ }^{14} \mathrm{C} /{ }^{3} \mathrm{H}$-leucine & Acute \& chronic & Rat liver, rat liver slices & {$[2,83,94,100,106-108]$} \\
${ }^{14} \mathrm{C}$-glucosamine & Acute \& chronic & Rat liver slices, isolated hepatocytes & {$[16,81-83,94,100]$} \\
${ }^{14} \mathrm{C} /{ }^{3} \mathrm{H}$-fucose & Acute \& chronic & Rat liver, rat liver slices & {$[82,94,100,106-108]$} \\
${ }^{14} \mathrm{C}$-galactose & Acute & Rat liver slices & {$[100]$} \\
VLDL & Acute \& chronic & Rat liver, isolated hepatocytes & {$[27,37,40,104]$} \\
Triglycerides & Acute & Isolated hepatocytes & {$[1,111]$} \\
Albumin & Chronic & Rat liver, rat liver slices, precision cut liver slices, WIF-B & {$[2,35,38,94]$} \\
Transferrin & Chronic & Rat liver, human alcoholic liver & {$[2,47-49]$} \\
Hepatic triglyceride lipase & Chronic & Isolated hepatocytes & {$[67]$} \\
IGF-1 & Acute \& chronic & Isolated hepatocytes, rat liver slices & {$[64,113]$} \\
\hline
\end{tabular}

Table 2 Ethanol-induced defects in basolateral membrane delivery

\begin{tabular}{llll}
\hline Protein/label & EtOH exposure & System & References \\
\hline${ }^{14} \mathrm{C}-$ fucose & Acute & Rat liver & {$[46,96]$} \\
${ }^{3} \mathrm{H}-\mathrm{N}$-acetyl mannosamine & Acute & Rat liver & {$[46,96]$} \\
Glucagon receptor & Chronic & Rat liver & {$[41]$} \\
ASGP-R & Chronic & Rat liver & {$[11,12]$} \\
EGF-R & Chronic & Isolated hepatocytes & {$[18]$} \\
\hline
\end{tabular}

secretion from the basolateral surface. In general, proteins destined for the secretory pathway encode a signal sequence that directs their docking and cotranslational entry into the endoplasmic reticulum (ER). During transit through the ER, secretory proteins are step-wise glycosylated, properly folded and eventually packaged into coatomer II-coated vesicles for delivery to the cis-Golgi. During transit through the Golgi, the secretory proteins are further modified (e.g., sulfation or phosphorylation) and their carbohydrates further trimmed and modified. In the trans-Golgi network (TGN), the newly synthesized proteins are terminally glycosylated by the addition of $\mathrm{N}$-acetylglucosamine, galactose, sialic acid and/or fucose moieties. While the mechanistic details regulating sorting at the TGN are not fully understood, secreted proteins are packaged into discrete vesicles, delivered to the basolateral surface and released [75]. In general, surface delivery of secretory proteins is a microtubule-dependent process that is mediated by a host of other molecules [20, 53, 69, 72]. In this section, we will describe alcohol-induced defects in bulk secretion and in the secretion of specific molecules.

\section{Bulk secretion}

To investigate ethanol's effects on protein secretion, early studies examined bulk secretion of radiolabeled leucine incorporated into newly synthesized proteins in both the rat liver and liver slices [2, 83, 94, 100, 106-108]. Both acute and chronic ethanol exposure induced a significant decrease in secreted levels of ${ }^{14} \mathrm{C} /{ }^{3} \mathrm{H}$-leucine-labeled proteins $(35-40 \%)$ in both systems (Table 1). This decrease in secretion correlated with a reciprocal increase in hepatic protein content indicating that the alcohol-induced secretion defect was not due to altered protein synthesis. From studies using differential centrifugation to isolate intracellular organelles, it was further determined that the bulk of hepatic secretory proteins was retained in the Golgi in ethanol-treated hepatocytes suggesting a block in vesicle delivery from the TGN to the basolateral surface (Fig. 1) [108].

To further pinpoint where the block in secretion was occurring, newly synthesized proteins were also labeled with ${ }^{14} \mathrm{C}$-glucosamine (added in the ER), ${ }^{14} \mathrm{C} /{ }^{3} \mathrm{H}$-fucose or ${ }^{14} \mathrm{C}$-galactose (both added in the TGN) [16, 81-83, 94, 100, 106-108]. A similar impairment in secretion was seen for all three labels along with a corresponding increase in intracellular protein content. Since the majority of ${ }^{14} \mathrm{C} /{ }^{3} \mathrm{H}$ fucose-labeled proteins accumulated in the Golgi and there were no changes in total glycosylation, the authors concluded that alcohol impaired post-Golgi sorting and vesicle delivery [16, 107, 108]. Additional studies performed in isolated hepatocytes [3] confirmed these results indicating that the ethanol-induced impairment of bulk secretion was highly reproducible and independent of the system used (Table 1). 


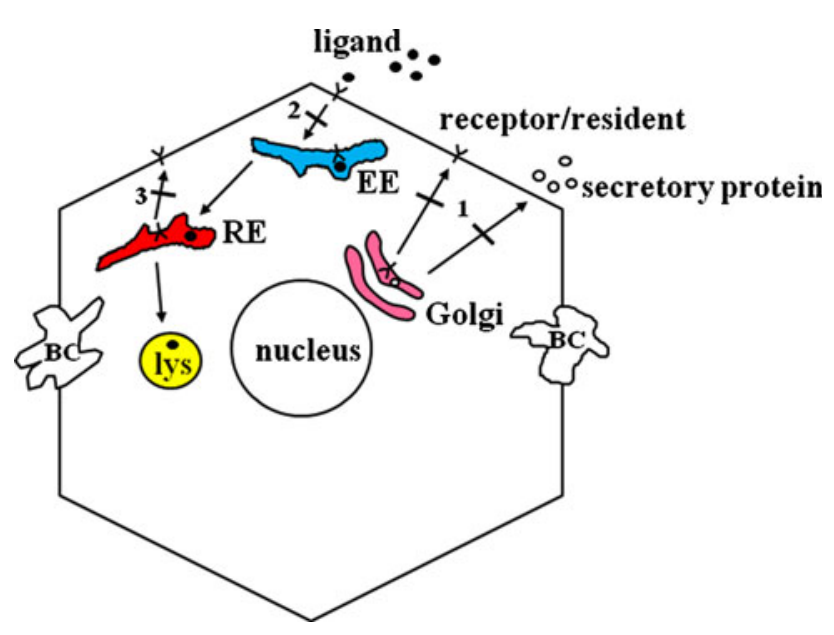

Fig. 1 Alcohol-induced defects in hepatic trafficking. Secretion and delivery of newly synthesized basolateral proteins is impaired in ethanol-exposed hepatocytes (step 1). Also, three major steps in the itineraries of various receptors are impaired by ethanol metabolism: transport of newly synthesized receptors from the Golgi to the basolateral membrane (step 1); receptor internalization from the plasma membrane (step 2); and recycling of receptors from recycling endosomes (RE) back to the cell surface (step 3). $E E$ early endosome; $R E$ recycling endosome; lys lysosome; $B C$ bile canaliculus

Addition of acetaldehyde also led to impaired hepatic secretion indicating that ethanol metabolism is required for the defect [106]. This is consistent with findings where addition of cyanamide (an aldehyde dehydrogenase (ALDH) inhibitor that leads to increased acetaldehyde levels) potentiated the impairment, while 4-methyl pyrazole (4-MP) (an alcohol dehydrogenase (ADH) inhibitor that leads to decreased acetaldehyde levels) prevented the observed secretion defects [83, 106, 107]. Together, these early studies indicate that ethanol significantly impairs hepatic protein secretion, and the defect is likely mediated by acetaldehyde.

\section{Albumin}

Serum albumin is a plasma protein that is synthesized and secreted in high quantities by hepatocytes. In the blood, albumin plays an important role in the maintenance of osmotic pressure and serves as a carrier for small hydrophobic molecules including hormones and unconjugated bilirubin [28]. Because decreased serum albumin levels can be associated with liver injury, it was of particular interest to directly determine whether alcohol exposure led to impaired albumin secretion. Chronic ethanol exposure in rats led to a significant increase in hepatic retention of both albumin and its precursor form, proalbumin, with a reciprocal decrease in secretion (Table 1) [2, 94]. More recent studies in both rat liver slices and polarized, hepatic WIF-B cells have also determined that ethanol exposure impairs albumin secretion (Table 1) [35, 38]. As for bulk secretion, addition of 4-MP to precision cut liver slices prevented the impairment, indicating that the defect requires ethanol metabolism and is likely mediated by acetaldehyde [38].

\section{Other secreted molecules}

Early findings in primary hepatocytes indicated that acute ethanol treatment also impairs ${ }^{14} \mathrm{C}$-glycerol labeled glycerolipid secretion that correlates with an increase in hepatic glycerolipid formation (triglycerides) (Table 1) [111]. Additional studies identified that both acute and chronic ethanol exposure impaired VLDL secretion in rat livers and primary hepatocytes [27, 37, 40, 104]. These findings correlated with an increase in hepatic triglycerides, suggesting a possible mechanism for the clinically observed fatty liver. Chronic ethanol treatment in isolated hepatocytes has also been shown to impair hepatic triglyceride lipase secretion, while both acute and chronic treatment impairs IGF-1 secretion, the latter of which is prevented by the addition of 4-MP [64, 67, 113]. Also, studies examining transferrin processing and secretion noted increased hepatic transferrin in the Golgi of both rats and humans with alcoholic liver disease [2, 47-49]. This correlated with decreased transferrin secretion and altered glycosylation, the latter of which serves as a marker for patients with alcoholic liver disease. Together, it is clear that ethanol induces significant defects in hepatic secretion (Table 1) and impaired secretion requires ethanol metabolism and is likely mediated by acetaldehyde.

Ethanol-induced alterations in plasma membrane composition

The basolateral plasma membrane is characterized by a specific complement of lipids and proteins not only required for membrane structure and integrity, but also for proper function. Although the details by which membrane proteins are sorted and delivered to the basolateral plasma membrane are still emerging, the newly synthesized membrane-bound glycoproteins are transported through the same biosynthetic compartments and are similarly glycosylated and processed as secretory proteins [20, 53, 69, 72]. Because of this similarity and the importance of this pathway, studies have examined the effects of ethanol on basolateral membrane delivery. In this section, we will briefly describe the known impairments in this process (Table 2).

\section{Bulk membrane delivery}

To investigate ethanol's effects on basolateral membrane biogenesis, early studies examined bulk delivery of newly synthesized ${ }^{14} \mathrm{C}$-fucose- or ${ }^{3} \mathrm{H}-\mathrm{N}$-acetyl mannosamine (a 
Table 3 Ethanol-induced defects in clathrin-mediated endocytosis

\begin{tabular}{llll}
\hline Protein & EtOH exposure & System & References \\
\hline ASGP & Acute \& Chronic & Isolated hepatocytes, intact perfused rat liver, HAD & {$[11,13,14,54,76,92,102]$} \\
ASGP-R & Acute \& Chronic & Isolated hepatocytes, WIF-B & {$[35,54,102]$} \\
Iron via transferrin & Acute & Isolated hepatocytes & {$[4]$} \\
Transferrin receptor & Chronic & WIF-B & {$[24]$} \\
Polymeric IgA receptor & Chronic & WIF-B & {$[24]$} \\
Chylomicron remnants & Chronic & Isolated hepatocytes & {$[39]$} \\
TGF- $\alpha$ & Chronic & Isolated hepatocytes & {$[99]$} \\
TNF- $\alpha$ & Chronic & Isolated hepatocytes & {$[99]$} \\
IL-6 & Chronic & Isolated hepatocytes & {$[99]$} \\
EGF & Chronic & Isolated hepatocytes & {$[10,18,63]$} \\
Insulin & Chronic & Isolated hepatocytes, humans & {$[62,71,91]$} \\
Growth hormone & Acute \& chronic & Rat liver slices, humans & {$[17,113]$} \\
Glucagon & Chronic & Rat liver plasma membranes & {$[41]$} \\
Aminopeptidase-N & Chronic & WIF-B & {$[35]$} \\
Dipeptidyl peptidase IV & Chronic & WIF-B & {$[24]$} \\
Hemagglutinin & Chronic & WIF-B & {$[24]$} \\
\hline
\end{tabular}

sialic acid precursor)-labeled glycoproteins [46, 96]. As for secretory proteins, the delivery of membrane glycoproteins was impaired in ethanol-treated hepatocytes with a reciprocal increase in intracellular protein content (Fig. 1) [46, 96]. To confirm that ethanol exposure altered basolateral membrane composition, concanavalin A (ConA) binding assays were performed [58]. Importantly, this lectin has a high affinity for glucose and mannose residues that are enriched on basolateral glycoproteins. Consistent with impaired glycoprotein delivery, less ConA surface labeling was observed for isolated hepatocytes from chronically fed animals [58] indicating altered plasma membrane composition. Also consistent with these results are the findings that decreased levels of newly synthesized receptors (Table 2) were detected at the plasma membrane in ethanol-exposed hepatocytes [11, 12, 18, 41]. Together, these results indicate that ethanol impairs basolateral delivery of glycoproteins; however, it is not yet known whether the defect requires ethanol metabolism, studies that are likely forthcoming.

\section{Ethanol-induced impairment of clathrin-mediated endocytosis}

Endocytosis serves as the interface between the cell and its external environment and is vital to many cellular processes including intercellular communication, nutrition, cell motility and signal transduction. Endocytosis includes several diverse mechanisms by which molecules are internalized at the plasma membrane and are packaged into discrete transport vesicles. There are at least three major internalization routes in mammalian cells: clathrin-mediated, caveolae/raft-mediated and non-clathrin/non-raft mediated $[15,21,52]$ that are characterized by specific molecular players, cargoes and regulators. In general, the ligands and receptors that display alcohol-induced internalization defects are internalized via clathrin-mediated pathways (Table 3). In contrast, internalization of markers of caveolae/raft-mediated and fluid phase endocytosis and non-vesicle-mediated uptake are not changed by ethanol exposure. In this section, we will list and discuss which ligands, receptors and other markers have been examined for defects in endocytosis.

\section{ASGP-R}

Much of our understanding of the alcohol-induced defects in endocytosis comes from studies examining the trafficking of asialoglycoproteins (ASGP) and their receptor, the ASGP receptor (ASGP-R), in control and ethanol treated hepatic cells (Fig. 1). After synthesis, ASGP-R is delivered to the basolateral plasma membrane where it is available to bind circulating ligands. Upon ligand binding, the receptorligand complex is internalized via clathrin-mediated endocytosis and is delivered to early endosomes. The ligand-receptor complex is then delivered to recycling endosomes where the ligand dissociates due to the acidic environment. The ligand is then transported to the lysosome for degradation, while the receptor recycles back to the basolateral plasma membrane for further rounds of ligand binding (Fig. 1). At least three steps in the itinerary of ASGP-R have been shown to be impaired by ethanol metabolism: transport of newly synthesized ASGP-R from 
the Golgi to the basolateral plasma membrane, receptor internalization from the basolateral plasma membrane, and recycling of the receptors from recycling endosomes back to the cell surface (Fig. 1) [reviewed in 54]. These phenomena have been observed in a number of model systems after chronic and acute ethanol exposure, including isolated rat hepatocytes, WIF-B cells, and intact perfused rat liver (Table 3) [11, 13, 14, 35, 54, 76, 102 and reviewed in 98]. Addition of 4-MP (an ADH inhibitor) prevented the endocytic defect in both HepG2 cells stably expressing alcohol dehydrogenase and in WIF-B cells indicating that the effect requires ethanol metabolism and is likely mediated by acetylaldehyde [14, 35].

Other well-characterized receptors

The clathrin-mediated internalization of a number of other well-characterized receptors has also been shown to be impaired by ethanol exposure (Table 3). For example, transferrin via the transferrin receptor has been shown to be impaired in isolated rat hepatocytes after acute ethanol treatment, while internalization of transferrin receptor has been shown to be impaired in WIF-B cells after chronic treatment (Table 3) [4, 24]. As for ASGP-R, addition of 4-MP to rat hepatocytes prevented impaired transferrin receptor internalization, suggesting that the defect requires ethanol metabolism. The internalization of two other known clathrin-mediated endocytosis markers, polymeric $\operatorname{IgA}$ receptor and chylomicron remnants via the LDLreceptor, was also shown to be impaired in ethanol-treated WIF-B cells or rat hepatocytes, respectively [24, 39]. Together, these results indicate that the well-described internalization defect of ASGP-R is not specific to that receptor, but rather is a general result of impaired clathrinmediated endocytosis (Fig. 1).

\section{Cytokines, growth factors and hormones}

The internalization and/or serum clearance of many cytokines, growth factors, and hormones has been shown to be impaired by ethanol consumption. The list so far includes: TGF- $\alpha$, TNF- $\alpha$, IL- 6 , EGF, and insulin (all in rat hepatocytes), growth hormone and insulin (in cirrhotic patients), and glucagon (in isolated rat liver plasma membranes) (Table 3) [10, 18, 62, 63, 71, 91, 99]. Remarkably, most of these molecules are known to be internalized by clathrinmediated endocytosis $[8,9,23,61,73,80,88,103,105$, 109]. At present, it is not well established whether the impaired internalization of these various ligands requires ethanol metabolism, but based on the receptor defects described earlier, the prediction is that it does. Together, these results suggest that it is not the receptors themselves that are altered by ethanol metabolism, but rather it is a general regulator of clathrin-mediated internalization that is altered.

Other internalization pathways are not impaired by ethanol exposure

In general, fluid-phase endocytosis and caveolae/lipid raftmediated internalization are not likely impaired by ethanol exposure. Although not as extensively studied as clathrininternalized receptors and ligands, there are a handful of studies that have examined markers of other internalization routes. For example, in both rat livers and WIF-B cells, the fluid phase internalization of Lucifer yellow was not changed by ethanol treatment [5, 24]. Similarly, the internalization of the GPI-anchored protein, 5'nucleotidase $\left(5^{\prime} \mathrm{NT}\right)$, and of the cholera toxin B subunit (markers for raft-mediated internalization) was not changed in ethanoltreated WIF-B cells [24, 35]. Furthermore, the non-vesicle, non-raft mediated internalization of fluorescein diacetate was not changed in ethanol-treated WIF-B cells [24]. Together, these results indicate that ethanol exposure selectively impairs clathrin-mediated endocytosis.

\section{Mechanisms responsible for impaired protein trafficking}

Ethanol is metabolized via two pathways: $\mathrm{ADH}$-mediated or cytochrome P450 2E1 (CYP 2E1)-mediated. ADHmediated metabolism results in the production of acetaldehyde, a highly reactive intermediate that can form stable, covalent modifications on other macromolecules [89]. CYP 2E1-mediated ethanol metabolism not only leads to the formation of acetaldehyde, but also to the formation of oxygen and hydroxyethyl radicals that in turn promote the formation of other highly reactive intermediates [89]. Like acetaldehyde, many of these CYP 2E1-generated byproducts can form stable, covalent modifications on proteins, lipids, DNA and other macromolecules [89]. Thus, one hypothesis for alcohol-induced hepatotoxicity is that the accumulated covalent modifications from chronic alcohol consumption disrupt the normal function of hepatic proteins, lipids and DNA leading to hepatocyte dysfunction and liver injury. More recently, it has become apparent that alcohol exposure induces protein covalent-modifications that are part of the natural repertoire. To date, these posttranslational modifications include increased methylation, phosphorylation and acetylation $[36,43,45,65,66,68$, 115]. In particular, numerous proteins have been identified that are hyperacetylated upon ethanol exposure, and this list is expanding rapidly [78]. As for adduct formation, it is not clear how increased acetylation is related to the progression of hepatotoxicity. In this section, we will discuss 
how modifications on tubulin or components of the clathrin machinery may contribute to alcohol-induced defects in protein trafficking.

\section{Ethanol-induced tubulin modifications}

Many proteins can be modified by acetaldehyde, including tubulin, actin, calmodulin, hemoglobin, hepatic enzymes and plasma proteins $[31,50,51,57,84,112]$. In general, acetaldehyde is thought to form stable adducts with the $\varepsilon$-amino group of lysine residues [93, 95, 97]. One of the best-studied target proteins for acetaldehyde adduction is $\alpha$-tubulin [97]. In vitro, this protein was found to be preferentially modified on a highly reactive lysine (probably lysine 394$)[85,86]$ in a time and concentration dependent manner $[32,33]$. In vitro polymerization assays using low acetaldehyde:tubulin dimer levels further revealed that adduction drastically impaired microtubule formation [34]. This impairment occurred at substoichiometric amounts of acetaldehyde $(0.2 \mathrm{~mol}$ acetaldehyde/mol tubulin) [79] suggesting that low levels of adduction can have far reaching effects on microtubule function. The defect in tubulin polymerization was also examined in isolated hepatocytes from alcohol-fed rats [114]. After removing nocodazole (a reversible, microtubule depolymerizing agent), microtubule regrowth was found to be significantly impaired in ethanol-treated hepatocytes consistent with impaired polymerization seen in vitro. Ethanol-treated WIF-B cells exhibited a similar tubulin phenotype where microtubule regrowth after nocodazole washout was impaired [36].

Steady state microtubules in alcohol-treated WIF-B cells were also found to be more stable and acetylated 2-3-fold more than in control cells [36]. This post-translational modification is characteristic of stable microtubule populations [110]. These results were confirmed in livers from ethanol-fed rats and in ethanol-treated liver slices indicating that the findings have physiologic importance [36]. Because microtubule hyperacetylation is prevented by 4-MP (in WIF-Bs and liver slices) and potentiated by cyanamide (in WIF-Bs), increased acetylation and stability require alcohol metabolism and are likely mediated by acetaldehyde. Thus, ethanol metabolism impairs tubulin polymerization, but once microtubules are formed they are hyperstabilized.

Because many vesicle transport steps are microtubule dependent, alterations in microtubule dynamics will likely contribute to defects in protein trafficking. Although the precise relationship between acetaldehyde modified-tubulin and defects in protein trafficking is not known, a link between tubulin acetylation and defects in protein trafficking is emerging. There is evidence that different microtubule populations (and/or their modifications) support specific protein trafficking steps [60]. Of particular interest are studies performed in WIF-B cells that used a novel microtubule depolymerizing drug, 201-F [70]. This drug specifically depolymerizes dynamic microtubules leaving only stable, acetylated polymers behind. In 201-Ftreated WIF-B cells, both secretion and transcytosis were impaired.

These results prompted us to test whether the defects in secretion and endocytosis observed in ethanol-treated cells can be explained by increased microtubule acetylation and stability. To do this, we examined the trafficking of selected proteins in WIF-B cells treated with ethanol or trichostatin A (TSA), a potent inhibitor of histone deacetylases (HDACs), including HDAC6. HDAC6 is the major tubulin deacetylase in liver and WIF-B cells $[26,77]$ and when inhibited with TSA increased microtubule acetylation and stability is observed in the absence of ethanol [35]. As shown in ethanol-treated cells, clathrin-mediated internalization of ASGP-R and APN was impaired in TSAtreated cells, while raft/caveolae-mediated internalization of the GPI-anchored protein, $5^{\prime} \mathrm{NT}$, was not changed [35]. Also as in ethanol-treated cells, albumin secretion was inhibited by TSA [35]. These results combined with the remarkable similarity of our findings in ethanol or TSA treated cells with 201-F-treated cells lead us to conclude that increased microtubule acetylation and stability explain, in part, the alcohol-induced defects in protein trafficking.

Ethanol-induced modifications of the clathrin machinery

Clathrin-mediated internalization is a highly regulated process involving multiple proteins [for reviews see 15, 21, $42,52,53]$. In general, clathrin triskelions are recruited to and assembled at regions of the plasma membrane enriched in $\mathrm{PIP}_{2}$. Adaptor protein (AP) complexes, such as AP2, are targeted to the membrane by their $\alpha$-adaptin subunits and interact directly with sorting signals on internalized proteins (usually containing di-leucine or tyrosine-based motifs) via their $\mu 2$ subunits. The large GTPase, dynamin, is thought to be recruited and assembled on the necks of coated pits where it likely mediates membrane fission and vesicle release. The released vesicles are rapidly uncoated allowing for coat recycling and vesicle fusion with its target organelle.

At present, the specific mechanisms responsible for ethanol-induced defects in clathrin-mediated internalization are not known. Because the clathrin-mediated internalization of many unrelated proteins is impaired in situ, in isolated hepatocytes and in ethanol-treated WIF-B cells (Table 3), it is likely that a universal regulator of clathrinmediated internalization is impaired, not the receptors 
themselves. This is consistent with recent findings where clathrin heavy chain accumulates at the basolateral membrane in discrete puncta in ethanol-treated WIF-B cells [24] suggesting impaired vesicle budding. Additionally, dynamin-2 was found to be less tightly associated with membranes in ethanol-treated cells, further suggesting that vesicle fission was impaired. However, it is not clear what promotes basolateral accumulation of clathrin heavy chain or decreased dynamin membrane associations. Because impaired ASGP-R clathrin-mediated internalization required ethanol metabolism and is likely mediated by acetaldehyde [35], an exciting possibility is that the molecular machinery that drives clathrin-mediated endocytosis is more prone to adduction by acetaldehyde or other reactive metabolites than the molecules regulating other internalization routes. Clearly, this exciting hypothesis requires more research.

A similar scenario may also evolve for proteins involved in vesicle budding and fission from the TGN. Basolateral resident proteins contain targeting information that likely promotes their recruitment into clathrin-coated vesicles at the TGN [72]. These basolateral targeting signals are similar to the signals required for clathrin-internalization and the majority are either tyrosine-based or contain a di-leucine motif [72]. Thus, one exciting possibility is that an alcohol-induced modification of the clathrin machinery also leads to defective vesicle fission at the TGN. Although constitutive secretory proteins are thought to bud from the TGN in different vesicle populations than transmembrane proteins [74], it is not yet clear what factors are required for their formation. Because constitutive secretion is also impaired in ethanol-treated hepatic cells, one possibility is that at least some components of the clathrin machinery are shared, and that these components are readily modified by reactive alcohol metabolites. Clearly, this is a fertile area of investigation for future research.

Another possibility is that key components of the clathrin machinery may be hyperacetylated upon ethanol exposure leading to impaired internalization. Although this hypothesis is untested, it is supported by the findings that actin and cortactin, two members of the clathrin machinery, are known to be hyperacetylated in the presence of ethanol [78]. Both of these proteins regulate late stages of clathrin coated vesicle budding from the plasma membrane and the TGN $[6,7]$. In general, cortactin is thought to promote actin polymerization at sites of vesicle formation and recruit dynamin to the necks of budding vesicles $[6,7]$. At present, the exact mechanism by which cortactin, actin and dynamin function to promote vesicle release is not yet completely elucidated. However, acetylation of cortactin is known to prevent its association with actin [116]. From these results, we propose that alcohol-induced hyperacetylation leads to decreased interactions between actin and cortactin such that cortactin is no longer recruited to sites of clathrin-vesicle formation, thereby inhibiting dynamin recruitment and subsequent vesicle fission. We are currently testing this exciting possibility.

\section{Clinical implications of impaired protein trafficking}

In this section, we describe the possible clinical relevance of alcohol-induced alterations in protein trafficking. We first describe possible ramifications of impaired secretion and basolateral delivery of newly synthesized membrane proteins. We end with discussing how alterations in clathrin-mediated internalization may explain the altered levels of circulating molecules in the alcoholic patient, and how impaired internalization might explain some alcoholinduced nutritional deficiencies.

Impaired secretion may be related to hepatomegaly and fatty liver

Defects in post-Golgi transport have been associated with hepatomegaly, a common phenotype of early alcoholic liver disease. In the alcohol-exposed liver, newly synthesized hepatic secretory proteins, lipoproteins and lipids accumulate in the Golgi, leading to increased cellular content. Furthermore, increased protein levels are exacerbated by alcohol-induced decreases in lysosomal and proteosomal protein degradation [22]. Based on these observations, the hypothesis is that the increased cellular protein and lipid content lead to increased hepatocyte volume $[90,92,98]$. This in turn leads to the clinically observed hepatocyte "ballooning" that may cause increased hepatic pressure or more serious liver injury. Furthermore, increased cellular lipoproteins and the decreased secretion of glycerolipids have also been correlated to increased hepatic triglyceride accumulation, a hallmark of ethanol-induced fatty liver, another early stage of alcohol-induced injury.

Defects in hepatic secretion may also be responsible for changes in the levels of circulating macromolecules in the alcoholic patient. As discussed earlier, hepatocytes are responsible for the synthesis and delivery of the majority of plasma proteins and lipoproteins. As summarized in Table 1, the secretion of many of these proteins is known to be impaired by ethanol exposure including albumin, transferrin and coagulation factors. Thus, defects in secretion may lead to altered blood buffering and blood volume, iron delivery to peripheral tissues and clotting defects. Similarly, impaired biliary delivery of newly synthesized glycoproteins has been reported in ethanol-fed rats, which in turn might explain, in part, decreased bile production observed in the alcoholic liver [44, 59]. 
Decreased bile production has further been implicated in impaired absorption of dietary lipids observed in the alcoholic patient [101].

Impaired basolateral delivery alters membrane composition and function

Impaired surface delivery of newly synthesized membrane glycoproteins and lipids ultimately leads to changes in the lipid and protein composition of the basolateral membrane. Such changes in membrane content may have serious ramifications not only on membrane integrity and permeability, but also on membrane function. For example, the failure to properly deliver cell surface transporters may have far-reaching implications on ion homeostasis. Similarly, the failure to properly deliver surface receptors could lead to harmful alterations in diverse cellular processes ranging from nutrition to signal transduction.

Impaired clathrin-mediated internalization likely alters numerous hepatic processes

Chronic alcohol consumption leads to many nutritional side effects. Much of this is due to the fact that an alcoholic often substitutes the calories from ethanol for the calories from food such that he/she becomes malnourished [44, 59]. This is further exacerbated by the decreased intestinal absorption of many compounds including dietary fats, fat soluble vitamins, essential amino acids, folic acid, glucose and minerals such as calcium, zinc, iron and magnesium [101]. In general, these defects are not likely due to impaired clathrin-mediated endocytosis. Most fat soluble vitamins associate with bile acid micelles and enter passively across the membrane, while most minerals are absorbed paracellularly or through specific transporters (e.g., copper and zinc). Thus, decreased absorption may be due to decreased bile production (for dietary fats and fatsoluble vitamins) or decreased paracellular permeability (for minerals). However, there are some nutritional deficiencies that might be explained by impaired clathrinmediated internalization that are described in this section. Furthermore, there are some interesting examples of molecules that are increased in the alcoholic's circulation, yet their biologic responses are diminished or absent. We propose that this paradox may also be explained by impaired clathrin-mediated internalization as described in the following paragraphs.

\section{Vitamin $B_{12}$ and fat soluble vitamins}

Many alcoholics display serious vitamin $\mathrm{B}_{12}$ deficiencies $[44,59]$. Unlike many other hydrophobic vitamins, $B_{12}$ is internalized via clathrin-mediated mechanisms in the intestine, liver and kidney [101]. In the small intestine, vitamin $B_{12}$ associates with intrinsic factor, a small glycoprotein synthesized and secreted by gastric parietal cells [101]. The $B_{12}$-intrinsic factor complex binds enterocytes via its receptor, cubilin, which in turn is complexed to the transmembrane protein, megalin [101]. The entire $\mathrm{B}_{12^{-}}$ intrinsic factor-cubilin-megalin complex is then internalized in clathrin-coated vesicles [101]. Ultimately, the $\mathrm{B}_{12}$ is secreted into the circulation coupled to transcobalamin II [101]. Peripheral tissues (mainly the liver and kidney) bind the $\mathrm{B}_{12}$-transcobalamin II complexes via transcobalamin II receptors, and the whole assembly is internalized in clathrin-coated vesicles. Thus, the vitamin $\mathrm{B}_{12}$ deficiencies seen in alcoholic patients may be explained, in part, by impaired clathrin-mediated endocytosis. This interesting hypothesis clearly requires further consideration.

Although vitamins A and D are absorbed passively across most cell membranes in association with lipid micelles, there is an interesting scavenge mechanism operating in kidney proximal tubules [101]. These vitamins are retrieved from the urine by association with surfaceassociated megalin. As described earlier for vitamin $B_{12}$, the megalin-A or -D complexes are internalized in clathrincoated vesicles. Thus, one interesting prediction is that this clathrin-mediated scavenge mechanism is impaired in chronic alcoholics. However, it remains to be determined whether ethanol consumption impairs clathrin-mediated endocytosis in other cell types.

\section{Cytokines and growth factors}

As summarized in Table 3, the internalization of a host of cytokines and growth factors is known to be impaired in ethanol-treated hepatic cells or in livers from ethanol-fed rats. Remarkably, the majority of these molecules are internalized by clathrin-mediated mechanisms. Furthermore, many of these growth factors and cytokines are present in increased amounts in the alcoholic patient's circulation, yet their biologic effects are diminished [44, 59]. One such interesting paradox has been described for signaling via the TNF- $\alpha$ and IL- 6 pathways [reviewed in 29, 30]. The circulating levels of each of these cytokines are increased in alcoholic patients and in ethanol-fed animals $[29,30]$ where they exert proinflammatory responses. However, both of these ligands are also known to promote hepatoprotective activities and are required for liver regeneration $[25,29,30,87]$. The puzzle is why the hepatoprotective and hepatomitogenic effects of TNF- $\alpha$ and IL- 6 are selectively lost in alcohol-treated hepatocytes despite their high circulating levels. One possible explanation for impaired hepatic signaling is defects in IL-6 and TNF- $\alpha$ receptor internalization. The simple hypothesis is that decreased cytokine retrieval leads to decreased 
signaling, and thus, decreased hepatoprotection. Because alcohol-induced defects in basolateral delivery of the newly synthesized receptors and receptor recycling also lead to decreased cell surface receptor numbers (Fig. 1) [54], another intriguing hypothesis is that one or both of these steps is impaired in the receptors' itineraries. Clearly, these hypotheses need to be tested for IL- 6 , TNF- $\alpha$ and other signaling molecules.

\section{$A S G P-R$ and apoptosis}

Apoptosis, or programmed cell death, is an irreversible process whereby cells respond to either intrinsic or extrinsic signals that initiate cascades that lead to cell death. Ultimately, the apoptotic cell is fragmented into apoptotic bodies that are then internalized and degraded by neighboring cells. Recent work has suggested that the surfaces of apoptotic bodies are enriched in desialated glycoproteins, and that ASGP-R binds these desialated carbohydrates and mediates apoptotic fragment internalization and degradation $[19,55]$. More recently, it has been discovered that increased apoptotic bodies are present in alcohol-exposed livers and that their internalization is impaired by $40-60 \%$ [19]. Thus, one interesting hypothesis is that alcohol-induced alterations in protein trafficking are responsible for the impaired clearance of apoptotic bodies. Furthermore, addition of apoptotic bodies to cultured Kupffer cells have been shown to stimulate secretion of the proinflammatory cytokines, TNF- $\alpha$ and IL-6 [56]. These results suggest that decreased apoptotic body clearance in livers promote an inflammatory response leading to enhanced liver injury, a hypothesis that will be interesting to test.

\section{Conclusion and future directions}

From the work summarized here, it is clear that chronic alcohol consumption leads to defects in hepatic protein trafficking. As described earlier, there is evidence that modification of tubulin and components of the clathrin machinery by reactive ethanol metabolites or post-translational modifications of the natural repertoire may explain the trafficking defects. The challenge that remains is twofold. First, the specific alcohol-induced adducts or modifications on tubulin and on clathrin coat components must be identified, confirmed and catalogued. Second, and perhaps more importantly, the cellular consequences of these modifications on hepatic protein trafficking must be defined. For example, does alcohol-induced tubulin adduction or hyperacetylation lead to altered binding of microtubule associated proteins and/or motors that in turn impairs vesicle motility? Does this impact secretion, membrane protein delivery and/or endocytosis? Similarly, do clathrin machinery modifications lead to altered coat assembly, receptor recruitment, vesicle formation and/or vesicle budding? Careful morphologic and biochemical methods will be needed to answer these very important questions. Once the specific mechanisms are defined, it will be important to determine the direct physiologic consequences of impaired protein trafficking in the alcoholic patient. For example, does impaired clathrin-mediated internalization lead to increased circulating cytokines and decreased vitamin $B_{12}$ absorption? Does this then lead to altered hepatic cytokine signaling and malnourishment in the alcoholic? It is also important to determine whether alcohol-induced defects in protein trafficking are specific to the hepatocyte or are a more general phenomenon observed in other cells of the liver and in other organs. Clearly, these hypotheses are in nascent form, and much is left to be done to test them. Continued research in this emerging field will not only increase our understanding of the pathogenesis of alcoholic liver disease, but may also provide novel therapeutic approaches to treatment.

Conflict of interest statement The authors report no conflicts of interest.

\section{References}

1. Annable W, Cooper C (1974) Inhibition of release of hepatic triglyceride by ethanol-a reappraisal. Biochem Pharmacol 23:2063-2068

2. Baraona E, Leo MA, Borowsky SA, Lieber CS (1977) Pathogenesis of alcohol-induced accumulation of protein in the liver. J Clin Invest 60:546-554

3. Baraona E, Pikkarainen P, Salaspuro M, Finkelman F, Lieber CS (1980) Acute effects of ethanol on hepatic protein synthesis and secretion in the rat. Gastroenterology 79:104-111

4. Beloqui O, Nunes RM, Blades B, Berk PD, Potter BJ (1986) Depression of iron uptake from transferrin by isolated hepatocytes in the presence of ethanol is a $\mathrm{pH}$-dependent consequence of ethanol metabolism. Alcohol Clin Exp Res 10:463-470

5. Camacho KB, Tuma DJ, Casey CA (1996) Zonal differences in ethanol-induced impairments in fluid-phase endocytosis in rat hepatocytes. Alcohol Clin Exp Res 20:589-594

6. Cao H, Orth JD, Chen J, Weller SG, Heuser JE, McNiven MA (2003) Cortactin is a component of clathrin-coated pits and participates in receptor-mediated endocytosis. Mol Cell Biol 23:2162-2170

7. Cao H, Weller S, Orth JD, Chen J, Huang B, Chen JL, Stamnes M, McNiven MA (2005) Actin and Arf1-dependent recruitment of a cortactin-dynamin complex to the Golgi regulates postGolgi transport. Nat Cell Biol 7:483-492

8. Carpentier JL (1992) Insulin-induced and constitutive internalization of the insulin receptor. Horm Res 38:13-18

9. Carpentier JL, Paccaud JP, Backer J, Gilbert A, Orci L, Kahn CR, Baecker J (1993) Two steps of insulin receptor internalization depend on different domains of the beta-subunit. J Cell Biol 122:1243-1252 
10. Casey CA, Camacho KB, Tuma DJ (1992) The effects of chronic ethanol administration on the rates of internalization of various ligands during hepatic endocytosis. Biochim Biophys Acta 1134:96-104

11. Casey CA, Kragskow SL, Sorrell MF, Tuma DJ (1987) Chronic ethanol administration impairs the binding and endocytosis of asialo-orosomucoid in isolated hepatocytes. J Biol Chem 262:2704-2710

12. Casey CA, Kragskow SL, Sorrell MF, Tuma DJ (1990) Effect of chronic ethanol administration on total asialoglycoprotein receptor content and intracellular processing of asialoorosomucoid in isolated rat hepatocytes. Biochim Biophys Acta 1052:18

13. Casey CA, Volentine GD, Jankovich CJ, Kragskow SL, Tuma DJ (1990) Effect of chronic ethanol administration on the uptake and degradation of asialoglycoproteins by the perfused rat liver. Biochem Pharmacol 40:1117-1123

14. Clemens DL, Halgard CM, Cole JR, Miles RM, Sorrell MF, Tuma DJ (1996) Impairment of the asialoglycoprotein receptor by ethanol oxidation. Biochem Pharmacol 52:1499-1505

15. Conner SD, Schmid SL (2003) Regulated portals of entry into the cell. Nature 422:37-44

16. Cottalasso D, Domenicotti C, Traverso N, Pronzato M, Nanni G (2002) Influence of chronic ethanol consumption on toxic effects of 1, 2-dichloroethane: glycolipoprotein retention and impairment of dolichol concentration in rat liver microsomes and Golgi apparatus. Toxicology 178:229-240

17. Cuneo RC, Hickman PE, Wallace JD, Teh BT, Ward G, Veldhuis JD, Waters MJ (1995) Altered endogenous growth hormone secretory kinetics and diurnal GH-binding protein profiles in adults with chronic liver disease. Clin Endocrinol (Oxf) 43:265-275

18. Dalke DD, Sorrell MF, Casey CA, Tuma DJ (1990) Chronic ethanol administration impairs receptor-mediated endocytosis of epidermal growth factor by rat hepatocytes. Hepatology 12:1085-1091

19. Dalton SR, Lee SM, King RN, Nanji AA, Kharbanda KK, Casey CA, McVicker BL (2009) Carbon tetrachloride-induced liver damage in asialoglycoprotein receptor-deficient mice. Biochem Pharmacol 77:1283-1290

20. De Matteis MA, Luini A (2008) Exiting the Golgi complex. Nat Rev Mol Cell Biol 9:273-284

21. Doherty GJ, McMahon HT (2009) Mechanisms of endocytosis. Annu Rev Biochem 78:857-902

22. Donohue TM Jr, Cederbaum AI, French SW, Barve S, Gao B, Osna NA (2007) Role of the proteasome in ethanol-induced liver pathology. Alcohol Clin Exp Res 31:1446-1459

23. Escobar GA, McIntyre RC Jr, Moore EE, Gamboni-Robertson F, Banerjee A (2006) Clathrin heavy chain is required for TNF-induced inflammatory signaling. Surgery 140:268-272

24. Fernandez DJ, McVicker BL, Tuma DJ, Tuma PL (2009) Ethanol selectively impairs clathrin-mediated internalization in polarized hepatic cells. Biochem Pharmacol 78:648-655

25. Gao B (2005) Cytokines, STATs and liver disease. Cell Mol Immunol 2:92-100

26. Grozinger CM, Hassig CA, Schreiber SL (1999) Three proteins define a class of human histone deacetylases related to yeast Hda1p. Proc Natl Acad Sci USA 96:4868-4873

27. Grunnet N, Jensen F, Kondrup J, Dich J (1985) Effect of ethanol on fatty acid metabolism in cultured hepatocytes: dependency on incubation time and fatty acid concentration. Alcohol 2:157161

28. Hankins J (2006) The role of albumin in fluid and electrolyte balance. J Infus Nurs 29:260-265

29. Hoek JB, Pastorino JG (2002) Ethanol, oxidative stress, and cytokine-induced liver cell injury. Alcohol 27:63-68
30. Hoek JB, Pastorino JG (2004) Cellular signaling mechanisms in alcohol-induced liver damage. Semin Liver Dis 24:257-272

31. Jennett RB, Saffari-Fard A, Sorrell MF, Smith SL, Tuma DJ (1989) Increased covalent binding of acetaldehyde to calmodulin in the presence of calcium. Life Sci 45:1461-1466

32. Jennett RB, Sorrell MF, Johnson EL, Tuma DJ (1987) Covalent binding of acetaldehyde to tubulin: evidence for preferential binding to the alpha-chain. Arch Biochem Biophys 256:10-18

33. Jennett RB, Sorrell MF, Saffari-Fard A, Ockner JL, Tuma DJ (1989) Preferential covalent binding of acetaldehyde to the alpha-chain of purified rat liver tubulin. Hepatology 9:57-62

34. Jennett RB, Tuma DJ, Sorrell MF (1980) Effect of ethanol and its metabolites on microtubule formation. Pharmacology 21:363-368

35. Joseph RA, Shepard BD, Kannarkat GT, Rutledge TM, Tuma DJ, Tuma PL (2008) Microtubule acetylation and stability may explain alcohol-induced alterations in hepatic protein trafficking. Hepatology 47:1745-1753

36. Kannarkat GT, Tuma DJ, Tuma PL (2006) Microtubules are more stable and more highly acetylated in ethanol-treated hepatic cells. J Hepatol 44:963-970

37. Kharbanda KK, Todero SL, Ward BW, Cannella III JJ, Tuma DJ (2009) Betaine administration corrects ethanol-induced defective VLDL secretion. Mol Cell Biochem 327:75-78

38. Klassen LW, Thiele GM, Duryee MJ, Schaffert CS, DeVeney AL, Hunter CD, Olinga P, Tuma DJ (2008) An in vitro method of alcoholic liver injury using precision-cut liver slices from rats. Biochem Pharmacol 76:426-436

39. Lakshmanan MR, Ezekiel M, Campbell BS, Muesing RA (1986) Binding, uptake, and metabolism of chylomicron remnants by hepatocytes from control and chronic ethanol-fed rats. Alcohol Clin Exp Res 10:412-418

40. Lakshmanan MR, Felver ME, Veech RL (1980) Alcohol and very low density lipoprotein synthesis and secretion by isolated hepatocytes. Alcohol Clin Exp Res 4:361-365

41. Lee H, Hosein EA (1982) Chronic alcohol feeding and its withdrawal on the structure and function of the rat liver plasma membrane: a study with 125I-labelled glucagon binding as a metabolic probe. Can J Physiol Pharmacol 60:1171-1176

42. Lee SM, Casey CA, McVicker BL (2009) Impact of asialoglycoprotein receptor deficiency on the development of liver injury. World J Gastroenterol 15:1194-1200

43. Lee YJ, Shukla SD (2007) Histone H3 phosphorylation at serine 10 and serine 28 is mediated by p38 MAPK in rat hepatocytes exposed to ethanol and acetaldehyde. Eur J Pharmacol 573:29-38

44. Lieber CS (2003) Relationships between nutrition, alcohol use, and liver disease. Alcohol Res Health 27:220-231

45. Lieber CS, Leo MA, Wang X, Decarli LM (2008) Effect of chronic alcohol consumption on Hepatic SIRT1 and PGC1alpha in rats. Biochem Biophys Res Commun 370:44-48

46. Mailliard ME, Sorrell MF, Volentine GD, Tuma DJ (1984) Impaired plasma membrane glycoprotein assembly in the liver following acute ethanol administration. Biochem Biophys Res Commun 123:951-958

47. Matsuda Y, Takada A, Sato H, Yasuhara M, Takase S (1985) Comparison between ballooned hepatocytes occurring in human alcoholic and nonalcoholic liver diseases. Alcohol Clin Exp Res 9:366-370

48. Matsuda Y, Takada A, Takase S, Sato H (1991) Accumulation of glycoprotein in the Golgi apparatus of hepatocytes in alcoholic liver injuries. Am J Gastroenterol 86:854-860

49. Matsuda Y, Takada A, Takase S, Yasuhara M (1991) Effects of ethanol on the secretion of hepatic secretory protein in rat alcoholic liver injury. Alcohol 8:433-437

50. Mauch TJ, Donohue TM Jr, Zetterman RK, Sorrell MF, Tuma DJ (1986) Covalent binding of acetaldehyde selectively inhibits 
the catalytic activity of lysine-dependent enzymes. Hepatology 6:263-269

51. Mauch TJ, Tuma DJ, Sorrell MF (1987) The binding of acetaldehyde to the active site of ribonuclease: alterations in catalytic activity and effects of phosphate. Alcohol Alcohol 22:103-112

52. Mayor S, Pagano RE (2007) Pathways of clathrin-independent endocytosis. Nat Rev Mol Cell Biol 8:603-612

53. McNiven MA, Thompson HM (2006) Vesicle formation at the plasma membrane and trans-Golgi network: the same but different. Science 313:1591-1594

54. McVicker BL, Casey CA (1999) Effects of ethanol on receptormediated endocytosis in the liver. Alcohol 19:255-260

55. McVicker BL, Tuma DJ, Casey CA (2007) Effect of ethanol on pro-apoptotic mechanisms in polarized hepatic cells. World $\mathrm{J}$ Gastroenterol 13:4960-4966

56. McVicker BL, Tuma DJ, Kharbanda KK, Kubik JL, Casey CA (2007) Effect of chronic ethanol administration on the in vitro production of proinflammatory cytokines by rat Kupffer cells in the presence of apoptotic cells. Alcohol Clin Exp Res 31:122-129

57. Medina VA, Donohue TM Jr, Sorrell MF, Tuma DJ (1985) Covalent binding of acetaldehyde to hepatic proteins during ethanol oxidation. J Lab Clin Med 105:5-10

58. Metcalf JP, Casey CA, Sorrell MF, Tuma DJ (1987) Chronic ethanol administration alters hepatic surface membranes as evidenced by decreased concanavalin A binding. Proc Soc Exp Biol Med 185:1-5

59. Mezey E (1980) Alcoholic liver disease: roles of alcohol and malnutrition. Am J Clin Nutr 33:2709-2718

60. Mizuno M, Singer SJ (1994) A possible role for stable microtubules in intracellular transport from the endoplasmic reticulum to the Golgi apparatus. J Cell Sci 107(Pt 5):1321-1331

61. Mosselmans R, Hepburn A, Dumont JE, Fiers W, Galand P (1988) Endocytic pathway of recombinant murine tumor necrosis factor in L-929 cells. J Immunol 141:3096-3100

62. Nygren A, Adner N, Sundblad L, Wiechel KL (1985) Insulin uptake by the human alcoholic cirrhotic liver. Metabolism $34: 48-52$

63. O'Rourke MF, Tuma DJ, Casey CA (1997) Decreased binding and autophosphorylation of the epidermal growth factor receptor in ethanol-fed rats. Biochem Pharmacol 53:1445-1450

64. Oh YI, Kim JH, Kang CW (2008) Effects of ethanol on insulinlike growth factor-I system in primary cultured rat hepatocytes: implications of JNK1/2 and alcoholdehydrogenase. World J Gastroenterol 14:4324-4331

65. Pal-Bhadra M, Bhadra U, Jackson DE, Mamatha L, Park PH, Shukla SD (2007) Distinct methylation patterns in histone H3 at Lys-4 and Lys-9 correlate with up- \& down-regulation of genes by ethanol in hepatocytes. Life Sci 81:979-987

66. Park PH, Miller R, Shukla SD (2003) Acetylation of histone H3 at lysine 9 by ethanol in rat hepatocytes. Biochem Biophys Res Commun 306:501-504

67. Parkes JG, Auerbach W, Goldberg DM (1990) Effect of alcohol on lipoprotein metabolism. II. Lipolytic activities and mixed function oxidases. Enzyme 43:47-55

68. Picklo MJ Sr (2008) Ethanol intoxication increases hepatic $\mathrm{N}$-lysyl protein acetylation. Biochem Biophys Res Commun 376:615-619

69. Ponnambalam S, Baldwin SA (2003) Constitutive protein secretion from the trans-Golgi network to the plasma membrane. Mol Membr Biol 20:129-139

70. Pous C, Chabin K, Drechou A, Barbot L, Phung-Koskas T, Settegrana C, Bourguet-Kondracki ML, Maurice M, Cassio D, Guyot M, Durand G (1998) Functional specialization of stable and dynamic microtubules in protein traffic in WIF-B cells. J Cell Biol 142:153-165
71. Rifkin RM, Todd WW, Toothaker DR, Sussman A, Trowbridge M, Draznin B (1983) Effects of in vivo and in vitro alcohol administration on insulin binding and glycogenesis in isolated rat hepatocytes. Ann Nutr Metab 27:313-319

72. Rodriguez-Boulan E, Musch A (2005) Protein sorting in the Golgi complex: shifting paradigms. Biochim Biophys Acta 1744:455-464

73. Sachse M, van Kerkhof P, Strous GJ, Klumperman J (2001) The ubiquitin-dependent endocytosis motif is required for efficient incorporation of growth hormone receptor in clathrin-coated pits, but not clathrin-coated lattices. J Cell Sci 114:3943-3952

74. Saucan L, Palade GE (1992) Differential colchicine effects on the transport of membrane and secretory proteins in rat hepatocytes in vivo: bipolar secretion of albumin. Hepatology 15:714-721

75. Saucan L, Palade GE (1994) Membrane and secretory proteins are transported from the Golgi complex to the sinusoidal plasmalemma of hepatocytes by distinct vesicular carriers. J Cell Biol 125:733-741

76. Sharma RJ, Grant DA (1986) A differential effect between the acute and chronic administration of ethanol on the endocytotic rate constant, ke, for the internalisation of asialoglycoproteins by hepatocytes. Biochim Biophys Acta 862:199-204

77. Shepard BD, Joseph RA, Kannarkat GT, Rutledge TM, Tuma DJ, Tuma PL (2008) Alcohol-induced alterations in hepatic microtubule dynamics can be explained by impaired histone deacetylase 6 function. Hepatology 48:1671-1679

78. Shepard BD, Tuma PL (2009) Alcohol-induced protein hyperacetylation: mechanisms and consequences. World J Gastroenterol 15:1219-1230

79. Smith SL, Jennett RB, Sorrell MF, Tuma DJ (1992) Substoichiometric inhibition of microtubule formation by acetaldehydetubulin adducts. Biochem Pharmacol 44:65-72

80. Sorkin A, Goh LK (2009) Endocytosis and intracellular trafficking of ErbBs. Exp Cell Res 315:683-696

81. Sorrel MF, Tuma DJ, Barak AJ (1977) Evidence that acetaldehyde irreversibly impairs glycoprotein metabolism in liver slices. Gastroenterology 73:1138-1141

82. Sorrell MF, Nauss JM, Donohue TM Jr, Tuma DJ (1983) Effects of chronic ethanol administration on hepatic glycoprotein secretion in the rat. Gastroenterology 84:580-586

83. Sorrell MF, Tuma DJ, Schafer EC, Barak AJ (1977) Role of acetaldehyde in the ethanol-induced impairment of glycoprotein metabolism in rat liver slices. Gastroenterology 73:137-144

84. Stevens VJ, Fantl WJ, Newman CB, Sims RV, Cerami A, Peterson CM (1981) Acetaldehyde adducts with hemoglobin. J Clin Invest 67:361-369

85. Szasz J, Burns R, Sternlicht H (1982) Effects of reductive methylation on microtubule assembly. Evidence for an essential amino group in the alpha-chain. J Biol Chem 257:3697-3704

86. Szasz J, Yaffe MB, Elzinga M, Blank GS, Sternlicht H (1986) Microtubule assembly is dependent on a cluster of basic residues in alpha-tubulin. Biochemistry 25:4572-4582

87. Taub R (2003) Hepatoprotection via the IL-6/Stat3 pathway. J Clin Invest 112:978-980

88. Thiel S, Behrmann I, Dittrich E, Muys L, Tavernier J, Wijdenes J, Heinrich PC, Graeve L (1998) Internalization of the interleukin 6 signal transducer gp130 does not require activation of the Jak/STAT pathway. Biochem J 330(Pt 1):47-54

89. Tuma DJ, Casey CA (2003) Dangerous byproducts of alcohol breakdown-focus on adducts. Alcohol Res Health 27:285290

90. Tuma DJ, Casey CA, Sorrell MF (1990) Effects of ethanol on hepatic protein trafficking: impairment of receptor-mediated endocytosis. Alcohol Alcohol 25:117-125 
91. Tuma DJ, Casey CA, Sorrell MF (1991) Chronic ethanolinduced impairments in receptor-mediated endocytosis of insulin in rat hepatocytes. Alcohol Clin Exp Res 15:808-813

92. Tuma DJ, Casey CA, Sorrell MF (1991) Effects of alcohol on hepatic protein metabolism and trafficking. Alcohol Alcohol Suppl 1:297-303

93. Tuma DJ, Hoffman T, Sorrell MF (1991) The chemistry of acetaldehyde-protein adducts. Alcohol Alcohol Suppl 1:271276

94. Tuma DJ, Jennett RB, Sorrell MF (1981) Effect of ethanol on the synthesis and secretion of hepatic secretory glycoproteins and albumin. Hepatology 1:590-598

95. Tuma DJ, Jennett RB, Sorrell MF (1987) The interaction of acetaldehyde with tubulin. Ann N Y Acad Sci 492:277-286

96. Tuma DJ, Mailliard ME, Casey CA, Volentine GD, Sorrell MF (1986) Ethanol-induced alterations of plasma membrane assembly in the liver. Biochim Biophys Acta 856:571-577

97. Tuma DJ, Smith SL, Sorrell MF (1991) Acetaldehyde and microtubules. Ann N Y Acad Sci 625:786-792

98. Tuma DJ, Sorrell MF (1988) Effects of ethanol on protein trafficking in the liver. Semin Liver Dis 8:69-80

99. Tuma DJ, Todero SL, Barak-Bernhagen M, Sorrell MF (1996) Effects of chronic ethanol administration on the endocytosis of cytokines by rat hepatocytes. Alcohol Clin Exp Res 20:579-583

100. Tuma DJ, Zetterman RK, Sorrell MF (1980) Inhibition of glycoprotein secretion by ethanol and acetaldehyde in rat liver slices. Biochem Pharmacol 29:35-38

101. Tuma PL, Hubbard AL (2003) Transcytosis: crossing cellular barriers. Physiol Rev 83:871-932

102. Tworek BL, Tuma DJ, Casey CA (1996) Decreased binding of asialoglycoproteins to hepatocytes from ethanol-fed rats. Consequence of both impaired synthesis and inactivation of the asialoglycoprotein receptor. J Biol Chem 271:2531-2538

103. van Kerkhof P, Sachse M, Klumperman J, Strous GJ (2001) Growth hormone receptor ubiquitination coincides with recruitment to clathrin-coated membrane domains. J Biol Chem 276:3778-3784

104. Venkatesan S, Ward RJ, Peters TJ (1988) Effect of chronic ethanol feeding on the hepatic secretion of very-low-density lipoproteins. Biochim Biophys Acta 960:61-66
105. Vieira AV, Lamaze C, Schmid SL (1996) Control of EGF receptor signaling by clathrin-mediated endocytosis. Science 274:2086-2089

106. Volentine GD, Ogden KA, Kortje DK, Tuma DJ, Sorrell MF (1987) Role of acetaldehyde in the ethanol-induced impairment of hepatic glycoprotein secretion in the rat in vivo. Hepatology $7: 490-495$

107. Volentine GD, Tuma DJ, Sorrell MF (1984) Acute effects of ethanol on hepatic glycoprotein secretion in the rat in vivo. Gastroenterology 86:225-229

108. Volentine GD, Tuma DJ, Sorrell MF (1986) Subcellular location of secretory proteins retained in the liver during the ethanolinduced inhibition of hepatic protein secretion in the rat. Gastroenterology 90:158-165

109. Watanabe J, Kanamura S, Asada-Kubota M, Kanai K, Oka M (1984) Receptor-mediated endocytosis of glucagon in isolated mouse hepatocytes. Anat Rec 210:557-567

110. Westermann S, Weber K (2003) Post-translational modifications regulate microtubule function. Nat Rev Mol Cell Biol 4:938947

111. Wood CK, Lamb RG (1979) The effect of ethanol on glycerolipid biosynthesis by primary monolayer cultures of adult rat hepatocytes. Biochim Biophys Acta 572:121-131

112. Xu DS, Jennett RB, Smith SL, Sorrell MF, Tuma DJ (1989) Covalent interactions of acetaldehyde with the actin/microfilament system. Alcohol Alcohol 24:281-289

113. Xu X, Ingram RL, Sonntag WE (1995) Ethanol suppresses growth hormone-mediated cellular responses in liver slices. Alcohol Clin Exp Res 19:1246-1251

114. Yoon Y, Torok N, Krueger E, Oswald B, McNiven MA (1998) Ethanol-induced alterations of the microtubule cytoskeleton in hepatocytes. Am J Physiol 274:G757-G766

115. You M, Liang X, Ajmo JM, Ness GC (2008) Involvement of mammalian sirtuin 1 in the action of ethanol in the liver. Am J Physiol Gastrointest Liver Physiol 294:G892-G898

116. Zhang X, Yuan Z, Zhang Y, Yong S, Salas-Burgos A, Koomen J, Olashaw N, Parsons JT, Yang XJ, Dent SR, Yao TP, Lane WS, Seto E (2007) HDAC6 modulates cell motility by altering the acetylation level of cortactin. Mol Cell 27:197-213 